\title{
Association of blood manganese level with diabetes and renal dysfunction: a cross-sectional study of the Korean general population
}

\author{
Eun Sil Koh ${ }^{1,2}$, Sung Jun Kim ${ }^{1,3}$, Hye Eun Yoon 1,3, Jong Hee Chung ${ }^{4}$, Sungjin Chung ${ }^{1,2}$, Cheol Whee Park, \\ Yoon Sik Chang ${ }^{1,2}$ and Seok Joon Shin ${ }^{1,3^{*}}$
}

\begin{abstract}
Background: The purpose of this study was to evaluate the association between blood manganese levels and the prevalence of chronic diseases in the Korean population.

Methods: This was a cross-sectional study based on the Korean National Health and Nutrition Examination Survey (KNAHNES). The study included 3996 participants 20 years of age or older whose blood manganese levels had been measured. The participants were also evaluated for the presence of five chronic diseases: diabetes, renal dysfunction, hypertension, ischemic heart disease, and stroke.

Results: Blood manganese levels were significantly lower in the diabetes group compared with the non-diabetes group $(1.26 \pm 0.02$ vs. $1.35 \pm 0.01 \mu \mathrm{g} / \mathrm{dL} ; p=0.001)$ and the renal dysfunction group compared with those with normal renal function ( $1.28 \pm 0.03 \mathrm{vs} .1 .35 \pm 0.01 \mu \mathrm{g} / \mathrm{dL} ; p=0.04)$. There was no significant association between blood manganese levels and the presence of ischemic heart disease or stroke. A multivariate logistic regression analysis adjusted for age, sex, and body mass index was performed; the odds ratio was 0.652 ( $95 \%$ Cl: 0.46-0.92) for diabetes and 0.589 (95\% Cl: 0.39-0.88) for renal dysfunction when comparing the higher quartiles (Q2-4) with the lowest quartile (Q1) of blood manganese level. The prevalence of diabetes was 7.6\% in Q1 and 5.3\% in Q2-4 ( $p=0.02$ ). Similarly, the prevalence of renal dysfunction was $6.8 \%$ in Q1, compared with $4.6 \%$ in Q2-4 ( $p=0.02)$.

Conclusion: The prevalence of diabetes and renal dysfunction increased in participants with low blood manganese levels, suggesting that blood manganese may play a role in glucose homeostasis and renal function.
\end{abstract}

Keywords: Manganese, Diabetes, Renal dysfunction

\section{Background}

Manganese $(\mathrm{Mn})$ is an essential trace metal with insufficient intake in virtually all diets. It has been reported that $\mathrm{Mn}$ is involved in normal immune functions, regulation of blood sugar and cellular energy, and the defense mechanisms against free radicals [1,2]. Experimentally induced Mn deficiency caused a number of detrimental effects, such as impaired bone formation, abnormal glucose tolerance, low levels of high-density lipoprotein (HDL) cholesterol, and skin abnormalities in both animals and humans [3].

\footnotetext{
*Correspondence: imkidney@catholic.ac.kr

'Department of Internal Medicine, College of Medicine, The Catholic University of Korea, 222 Banpo-daero, Seoul 137-701, Republic of Korea ${ }^{3}$ Division of Nephrology, The Catholic University of Korea Incheon St. Mary's Hospital, 56, Dongsu-ro, Bupyeong-gu, Incheon 403-720, Republic of Korea Full list of author information is available at the end of the article
}

Conversely, excess occupational inhalation of Mn may be neurotoxic to humans, producing effects such as psychosis and Parkinsonism. Mn deficiency and intoxication are both associated with adverse metabolic and neuropsychiatric effects $[4,5]$. Nevertheless, little is known about the optimal blood Mn levels for maintaining homeostasis in humans.

One study found that $\mathrm{Mn}$ inhalation in adult animal models markedly down-regulated the gene expression of proteins critical to inflammatory responses or possessing pro-oxidant properties (e.g. tumor growth factor (TGF)- $\beta$ and neuronal nitric oxide synthase (nNOS)) [6]. These results suggested that a certain amount of Mn exposure may attenuate the inflammatory response to stressful environments. In contrast, $\mathrm{Mn}$ is an essential cofactor for metalloenzyme superoxide dismutase, which protects cells 
against antioxidant processes [3]. Malecki et al. demonstrated that Mn deficiency and the resultant decrease in manganese superoxide dismutase (MnSOD) activity caused heart mitochondria to be more sensitive to in vivo oxidative damage [7]. This study proposed that subclinical Mn deficiency might also contribute to excess oxidative stress.

Oxidative stress and inflammation are the main pathophysiology behind most chronic diseases. Therefore, we hypothesized that blood Mn levels could be related to the prevalence of chronic diseases. We conducted a cross-sectional analysis on the associations of blood $\mathrm{Mn}$ level with five chronic diseases (diabetes, renal dysfunction, hypertension, ischemic heart disease, and stroke) in a representative sample of the adult Korean population from the Korean National Health and Nutritional Examination Survey (KNHANES).

\section{Methods}

\section{Study population}

The KNHANES was performed to examine the general health and nutritional status of the civilian, noninstitutionalized Korean population. KNHANES is a cross-sectional and nationally representative survey composed of a health questionnaire, a health examination, and a nutrition survey. The fourth KNHANES (KNHANES IV) was conducted from 2007 to 2009 using a stratified, multistage probability sampling design. We used data from the second (2008) to third year (2009) of the KNHANES IV. Of the 8641 individuals who participated during this time, we analyzed those who were 20 years of age or older and tested for blood Mn. We excluded participants who were pregnant or had missing values and yielded a final sample size of 3996 adults. The Catholic University of Korea Incheon St. Mary's Hospital Institutional Review Board and the Korea Centers for Disease Control and Prevention approved the study protocol, and written informed consent was obtained from all participants before the study began.

\section{Laboratory measurements}

Blood Mn was measured using whole blood at the Neodin Medical Institute (certified by the Korean Ministry of Health and Welfare) in Seoul, Korea, following a standardized protocol. Blood Mn was analyzed by graphite furnace atomic absorption spectrometry with Zeeman background correction (Perkin Elmer AAS800, Perkin Elmer, Turku, Finland). The limit of detection was 0.016 $\mu \mathrm{g} / \mathrm{dL}$ for blood Mn. For internal quality assurance and control, standard reference materials were obtained from Bio-Rad (Lyphochek ${ }^{\circ}$ Whole Blood Metals Control). The inter-assay coefficients of variation ranged from $0.95 \%$ to $4.82 \%$ for blood Mn samples (reference values were $0.98,1.18,2.46$, and $3.28 \mu \mathrm{g} / \mathrm{dL}$ ). During the survey, overnight fasting venous blood samples were collected. The collected blood samples were properly processed, refrigerated, and transported in cold storage to the Neodin Medical Institute in Seoul, Korea.

\section{Chronic diseases}

Diabetes was defined as having one of the following: a fasting blood glucose $\geq 126 \mathrm{mg} / \mathrm{dL}$, a self-reported physician's diagnosis, medication use, or insulin administration at the time of interview. Serum creatinine level was measured using a Hitachi Automatic Analyzer 7600 (Hitachi, Japan) and a modified kinetic Jaffe reaction. The level of kidney function was attained using an abbreviated equation developed from the data from the Chronic Kidney Disease-Epidemiologic Collaboration Group (CKD-EPI) study to estimate the glomerular filtration rate (GFR) [8]. We defined renal dysfunction as an estimated GFR (eGFR) of $<65 \mathrm{~mL} / \mathrm{min} / 1.73 \mathrm{~m}^{2}$ [9]. Hypertension was defined as having one of the following: a mean systolic blood pressure of $\geq 140 \mathrm{mmHg}$, a mean diastolic blood pressure of $\geq$ $90 \mathrm{mmHg}$, a self-reported physician's diagnosis, or antihypertensive medication use at the time of interview. Ischemic heart disease and stroke were based on a self-reported physician's diagnosis.

\section{Other variables}

Information on age, sex, residential area, educational status, smoking exposure and alcohol consumption, occupation, body mass index (BMI), and nutritional intake were based on a health questionnaire. Residential area was categorized as either urban or rural. Seoul (the capital city of South Korea) and six other metropolitan cities were grouped as urban areas, and the remaining regions were defined as rural areas. Educational status was divided into $\geq$ college or $\leq$ high school. Alcohol consumption was indicated as positive for participants who had consumed at least $30 \mathrm{~g}$ per day over the last year. The participants' occupations were categorized as services (which included students, housewives, and the unemployed), agriculture, fishery, or industry. BMI was calculated as weight in kilograms divided by height in meters squared.

\section{Statistical analysis}

All statistical analyses and calculations were performed using SAS V9.2 (SAS Institute). We used the stratification variables and sampling weights designated by the Korean Centers for Disease Control and Prevention.

The baseline characteristics were presented as mean \pm standard error (SE), median and range, or frequency and proportions. Comparisons of each variable between the two groups were performed using Student's $t$-test or modified Rao-Scott chi-square test. Demographic characteristics were analyzed according to eGFR $(<65$ or $\geq$ 
$65 \mathrm{~mL} / \mathrm{min} / 1.73 \mathrm{~m}^{2}$ ), the presence of chronic diseases (diabetes, hypertension, ischemic heart disease, or stroke), and quartiles of blood Mn levels (first lowest quartile vs. other quartiles). The odds ratios (OR) and $95 \%$ confidence intervals $(95 \% \mathrm{CI})$ of the associated factors for reduced eGFR and blood Mn quartiles were estimated using logistic regression. A $p$-value of $<0.05$ was considered statistically significant.

\section{Results}

The total number of participants was 3996, and the weighted number was $36,990,120$. The mean participant age was $45 \pm 0.2$ years. The mean blood Mn level in the Korean adult population was $1.34 \pm 0.01 \mu \mathrm{g} / \mathrm{dL}$, and the mean eGFR as calculated by the CKD-EPI equation was $95.1 \mathrm{ml} / \mathrm{min} / 1.73 \mathrm{~m}^{2}$. The mean systolic blood, diastolic blood, and arterial pressures were $116.9 \pm 0.4 \mathrm{mmHg}$, $76.7 \pm 0.3 \mathrm{mmHg}$ and $88.7 \pm 0.2 \mathrm{mmHg}$, respectively. The baseline clinical characteristics of the participants were shown in Table 1. Blood Mn levels were significantly different in the baseline characteristics of the following variables: sex, smoking exposure, the history of alcohol drinking, and residential area (data not shown). Mn levels were also different according to the presence of chronic disease (Table 2). Participants with diabetes had significantly lower blood Mn levels than those without diabetes $(p<0.05)$. Renal dysfunction and hypertension presented similarly $(p<0.05)$. There was no significant difference in the blood Mn levels of participants with or without ischemic heart disease or stroke.

We also evaluated the association of blood Mn level with eGFR and blood pressure. In a linear regression analysis adjusted for age, sex, and BMI, there was no statistically significant association between blood $\mathrm{Mn}$ levels and eGFR. However, blood Mn levels were positively associated with systolic blood $(\beta$ coefficient $=1.52$, $p=0.01$ ), diastolic ( $\beta$ coefficient $=1.01, p=0.02$ ), and mean arterial blood pressures ( $\beta$ coefficient $=1.26, p=0.004$ ) after adjusting for age, sex, BMI, and presence of diabetes.

We divided blood Mn level into quartiles (Q1-Q4) and analyzed the risk for chronic disease. In a multivariate logistic regression analysis, after adjusting for age, sex, and BMI, participants with blood Mn levels in the higher quartiles had a tendency to have lower odds ratios (OR) for the presence of diabetes with a significant test for trend $(p=$ 0.02; Table 3). When the higher quartiles $(\mathrm{Q} 2-4$, blood $\mathrm{Mn} \geq 1.060 \mu \mathrm{g} / \mathrm{dL}$ ) were compared with the lowest quartile (Q1, blood Mn $\leq 1.059 \mu \mathrm{g} / \mathrm{dL}$ ), the OR for diabetes was 0.652 (95\% CI: $0.46-0.92$ ) and 0.589 for renal dysfunction (95\% CI: $0.39-0.88, p<0.05$ ) (Table 4). It was well known that diabetes and hypertension were significant risk factors for renal dysfunction; therefore, when adjusted for the presence of diabetes and hypertension, the OR for renal dysfunction was 0.617 (95\% CI: 0.41-0.92, $p<0.05$ ).
Table 1 Demographic and clinical characteristics of participants $(n=3996)^{a}$

\begin{tabular}{lc}
\hline Characteristics & $\%($ SE) \\
\hline Age, $y^{\mathrm{b}}$ & $45.2 \pm 0.2$ \\
$<65$ & $86.2(0.6)$ \\
$\geq 65$ & $13.8(0.6)$ \\
Sex & \\
Male & $49.5(0.5)$ \\
Female & $50.5(0.5)$ \\
BMI, kg/m ${ }^{2}$ & \\
$<25$ & $68.9(0.9)$ \\
$\geq 25$ & $31.1(0.9)$ \\
Smoking exposure & \\
Never & $74.3(0.7)$ \\
Former or current & $25.7(0.7)$ \\
Alcohol drinking, g/day & \\
$<30$ & $90.1(0.6)$ \\
$\geq 30$ & $9.9(0.6)$ \\
Residence area & \\
Rural & $20.2(1.8)$ \\
Urban & $79.8(1.8)$
\end{tabular}

Education

$\leq$ High school $\quad 70.4(1.0)$

$\geq$ College $\quad 29.6(1.0)$

Occupation

Agriculture \& fishery $\quad 5.6$ (0.6)

$\begin{array}{ll}\text { Industry } & 9.2(0.5)\end{array}$

$\begin{array}{ll}\text { Services } & 85.3(0.8)\end{array}$

$\begin{array}{ll}\text { GFR, } \mathrm{ml} / \mathrm{min} / 1.73 \mathrm{~m}^{2 \mathrm{~b}} & 95.1 \pm 0.5\end{array}$

Blood pressure ${ }^{b}$

$\begin{array}{ll}\mathrm{SBP}, \mathrm{mmHg} & 116.9 \pm 0.4\end{array}$

$\begin{array}{ll}\mathrm{DBP}, \mathrm{mmHg} & 76.7 \pm 0.3\end{array}$

$\begin{array}{ll}\text { MAP, } \mathrm{mmHg} & 88.7 \pm 0.2\end{array}$

$\mathrm{BMI}=$ body mass index; GFR = glomerular filtration rate; $\mathrm{SBP}=$ systolic blood pressure; $\mathrm{DBP}=$ diastolic blood pressure; $\mathrm{MAP}=$ mean arterial pressure.

${ }^{\text {aData }}$ are given as\% (SE) unless otherwise indicated.

${ }^{b}$ Data as the mean $\pm \mathrm{SE}$.

The prevalence of diabetes was significantly higher in the lowest quartile (Q1) when compared with the higher quartiles Q2-4 (7.6\% vs. 5.3\%, $p=0.02)$. Similarly, the prevalence of renal dysfunction showed a significantly higher value in the lowest quartile (Q1) than in the higher quartiles $(6.8 \%$ vs. $4.6 \%, p=0.02$; Table 4$)$.

\section{Discussion}

This study was the first to examine the effects of blood Mn on chronic diseases in the general population and determine that blood Mn levels affected the morbidity of chronic diseases. It was found that low blood Mn levels 
Table 2 Characteristics of participants and blood Mn levels according to the presence of chronic diseases ${ }^{\mathrm{a}}$

\begin{tabular}{|c|c|c|c|c|c|c|c|c|c|c|c|c|c|c|c|}
\hline & \multicolumn{3}{|c|}{ Diabetes } & \multicolumn{3}{|c|}{ Renal dysfunction } & \multicolumn{3}{|c|}{ Hypertension } & \multicolumn{3}{|c|}{ IHD } & \multicolumn{3}{|c|}{ Stroke } \\
\hline & $\begin{array}{c}\text { Yes } \\
(n=255)\end{array}$ & $\begin{array}{c}\text { No } \\
(n=3735)\end{array}$ & $P$ value & $\begin{array}{c}\text { Yes } \\
(n=200)\end{array}$ & $\begin{array}{c}\text { No } \\
(n=3796)\end{array}$ & $P$ value & $\begin{array}{c}\text { Yes } \\
(n=1056)\end{array}$ & $\begin{array}{c}\text { No } \\
(n=2921)\end{array}$ & $P$ value & $\begin{array}{c}\text { Yes } \\
(n=49)\end{array}$ & $\begin{array}{c}\text { No } \\
(n=3941)\end{array}$ & $P$ value & $\begin{array}{c}\text { Yes } \\
(n=43)\end{array}$ & $\begin{array}{c}\text { No } \\
(\mathrm{n}=3947)\end{array}$ & $P$ value \\
\hline$\overline{\text { Age }(\geq 65 \mathrm{yrs})}$ & $39.1(3.7)$ & $12.2(0.6)$ & $<.001$ & $61.6(4.0)$ & $11.2(0.5)$ & $<.001$ & $30.8(1.6)$ & $7.7(0.6)$ & $<.001$ & $48.7(8.8)$ & $13.3(0.6)$ & $<.001$ & $42.8(9.2)$ & $13.5(0.6)$ & $\overline{<.001}$ \\
\hline Sex (male) & $46.0(3.4)$ & $49.7(0.6)$ & 0.31 & $46.4(3.6)$ & $49.7(0.5)$ & 0.38 & $55.0(1.5)$ & $47.4(0.7)$ & $<.001$ & $54.6(8.0)$ & $49.4(0.5)$ & 0.52 & $62.0(9.4)$ & $49.3(0.5)$ & 0.19 \\
\hline BMI $(\geq 25$ kg/m²) & 49.7 (3.6) & $29.9(0.9)$ & $<.001$ & $47.4(4.2)$ & $30.2(0.9)$ & $<.001$ & $46.2(1.7)$ & $25.8(0.9)$ & $<.001$ & $49.6(8.0)$ & $30.9(0.9)$ & 0.01 & $35.1(8.3)$ & $31.1(0.9)$ & 0.62 \\
\hline Smoking exposure & $23.0(2.8)$ & $25.8(0.8)$ & 0.34 & $9.2(2.2)$ & $26.6(0.8)$ & $<.001$ & $24.9(1.5)$ & $26.0(0.9)$ & 0.54 & $17.3(7.7)$ & $25.8(0.7)$ & 0.92 & $21.3(6.5)$ & $25.7(0.7)$ & 0.53 \\
\hline Alcohol ( $\geq 30$ g/day) & $6.3(1.7)$ & $10.2(0.6)$ & 0.08 & $5.7(1.9)$ & $10.2(0.6)$ & 0.07 & $13.5(1.3)$ & $8.7(0.6)$ & $<.001$ & $2.1(2.1)$ & $10.1(0.6)$ & 0.07 & $2.8(2.8)$ & $10.0(0.6)$ & 0.16 \\
\hline Residence (rural) & $20.6(3.4)$ & $20.2(1.8)$ & 0.89 & $20.9(4.1)$ & $20.1(1.8)$ & 0.83 & $22.2(2.2)$ & $19.5(1.8)$ & 0.07 & $29.2(7.7)$ & $20.1(1.8)$ & 0.17 & $24.0(6.7)$ & $20.2(1.8)$ & 0.54 \\
\hline Education ( $\geq$ college) & $16.4(2.5)$ & $30.4(1)$ & $<.001$ & $9.3(2.3)$ & $30.7(1.0)$ & $<.001$ & $18.7(1.5)$ & $33.5(1.1)$ & $<.001$ & $12.1(5.3)$ & $29.8(1.0)$ & 0.02 & $3.7(2.5)$ & $29.8(1.0)$ & $<.001$ \\
\hline Occupation & & & 0.02 & & & 0.93 & & & $<.001$ & & & $<.001$ & & & 0.95 \\
\hline Services \& others & $6.1(1.8)$ & $5.6(0.6)$ & & $5.2(1.6)$ & $5.6(0.6)$ & & $7.4(1.1)$ & $5.0(0.6)$ & & $19.0(7.1)$ & $5.4(0.6)$ & & $5.9(3.4)$ & $5.6(0.6)$ & \\
\hline Industry & $15.2(3.1)$ & $8.8(0.5)$ & & $8.6(2.2)$ & $9.2(0.5)$ & & $11.7(1.1)$ & $8.2(0.6)$ & & $4.2(2.7)$ & $9.2(0.5)$ & & $7.9(4.4)$ & $9.2(0.5)$ & \\
\hline Agriculture \& fishery & $78.8(3.4)$ & $85.6(0.8)$ & & $86.2(2.7)$ & $85.2(0.8)$ & & $81.0(1.5)$ & $86.8(0.8)$ & & $76.8(7.3)$ & $85.3(0.8)$ & & $86.2(5.3)$ & $85.2(0.8)$ & \\
\hline Blood Mn $(\mu \mathrm{g} / \mathrm{dL})^{b}$ & $1.26 \pm 0.02$ & $1.35 \pm 0.01$ & 0.001 & $1.28 \pm 0.03$ & $1.35 \pm 0.01$ & 0.04 & $1.32 \pm 0.01$ & $1.35 \pm 0.01$ & 0.04 & $1.25 \pm 0.05$ & $1.34 \pm 0.01$ & 0.09 & $1.35 \pm 0.08$ & $1.34 \pm 0.01$ & 0.94 \\
\hline
\end{tabular}

$\mathrm{HDD}=$ ischemic heart disease; $\mathrm{BMI}=$ body mass index; $\mathrm{Mn}=$ Manganese.

${ }^{a}$ Data are given as the percentage (SE) of each category in participants with or without chronic disease unless otherwise indicated. ${ }^{\mathrm{b}}$ Data as the mean $\pm \mathrm{SE}$. 
Table 3 Odds ratio and $95 \%$ confidence interval values of chronic disease presence with blood Mn level after adjusting covariates

\begin{tabular}{|c|c|c|c|c|c|c|c|c|c|c|}
\hline \multirow{2}{*}{$\begin{array}{c}\text { Mn level } \\
\text { quartile }(\mu \mathrm{g} / \mathrm{dL})\end{array}$} & \multicolumn{2}{|c|}{ Diabetes } & \multicolumn{2}{|c|}{ Renal dysfunction } & \multicolumn{2}{|c|}{ Hypertension } & \multicolumn{2}{|c|}{ IHD } & \multicolumn{2}{|c|}{ Stroke } \\
\hline & Unadjusted & Adjusted $^{a}$ & Unadjusted & Adjusted $^{\mathrm{b}}$ & Unadjusted & Adjusted $^{c}$ & Unadjusted & Adjusted $^{b}$ & Unadjusted & Adjusted $^{\mathbf{b}}$ \\
\hline $\mathrm{Q} 1(\leq 1.059)$ & 1 (reference) & 1 (reference) & 1 (reference) & 1 (reference) & 1 (reference) & 1 (reference) & 1 (reference) & 1 (reference) & 1 (reference) & 1 (reference) \\
\hline Q2 (1.060 - 1.277) & $0.66(0.45,0.97)$ & $0.65(0.43,0.99)$ & $0.52(0.34,0.81)$ & $0.51(0.31,0.84)$ & $1.09(0.86,1.37)$ & $1.21(0.93,1.57)$ & $1.03(0.41,2.57)$ & $1.04(0.38,2.80)$ & $0.92(0.37,2.30)$ & $0.91(0.35,2.39)$ \\
\hline Q3 (1.278 - 1.559) & $0.86(0.57,1.30)$ & $0.75(0.49,1.15)$ & $0.78(0.50,1.20)$ & $0.64(0.39,1.07)$ & $1.15(0.92,1.44)$ & $1.16(0.90,1.50)$ & $1.49(0.62,3.59)$ & $1.41(0.55,3.62)$ & $1.22(0.50,3.00)$ & $1.21(0.48,3.07)$ \\
\hline Q4 ( $\geq 1.560)$ & $0.53(0.35,0.80)$ & $0.53(0.34,0.84)$ & $0.67(0.43,1.05)$ & $0.72(0.43,1.20)$ & $0.86(0.69,1.07)$ & $1.07(0.83,1.39)$ & $0.47(0.17,1.36)$ & $0.55(0.18,1.65)$ & $0.94(0.30,2.94)$ & $1.22(0.39,3.80)$ \\
\hline$P$ value & 0.01 & 0.04 & 0.03 & 0.06 & 0.07 & 0.49 & 0.11 & 0.26 & 0.93 & 0.92 \\
\hline$P$ for trend & 0.02 & 0.02 & 0.22 & 0.28 & 0.28 & 0.65 & 0.41 & 0.60 & 0.94 & 0.62 \\
\hline
\end{tabular}

$\mathrm{HDD}=$ ischemic heart disease; $\mathrm{Mn}=$ manganese

adjusted for age, sex, body mass index and hypertension.

badjusted for age, sex, body mass index, diabetes and hypertension.

cadjusted for age, sex, body mass index and diabetes. 


\begin{tabular}{|c|c|c|c|c|c|}
\hline \multirow[t]{2}{*}{ Parameter } & \multicolumn{2}{|c|}{ Odds ratio ( $95 \%$ confidence interval) } & \multicolumn{3}{|c|}{ Prevalence $(\%, \mathrm{SE})$} \\
\hline & Unadjusted & Adjusted & Q1 & Q2-4 & $P$ value \\
\hline Diabetes & $0.68(0.50,0.94)$ & $0.65(0.46,0.92)^{b}$ & $7.6(0.9)$ & $5.3(0.5)$ & $0.02^{b}$ \\
\hline Renal dysfunction & $0.65(0.46,0.92)$ & $0.62(0.41,0.92)^{c}$ & $6.8(1.0)$ & $4.6(0.5)$ & $0.02^{c}$ \\
\hline Hypertension & $1.03(0.85,1.24)$ & $1.15(0.93,1.42)^{d}$ & $25.6(1.6)$ & $26.2(1.0)$ & $0.77^{d}$ \\
\hline IHD & $1.00(0.46,2.16)$ & $1.04(0.45,2.40)^{c}$ & $1.2(0.4)$ & $1.2(0.2)$ & $0.99^{c}$ \\
\hline Stroke & $1.03(0.48,2.20)$ & $1.11(0.51,2.42)^{c}$ & $0.8(0.2)$ & $0.8(0.2)$ & $0.94^{c}$ \\
\hline
\end{tabular}

$\mathrm{IHD}=$ ischemic heart disease.

ablood manganese quartiles ( $\mu \mathrm{g} / \mathrm{dL})$ : Q1 $\leq 1.059$, Q2 1.060 - 1.277, Q3 $1.278-1.559$, Q4 $\geq 1.560$

badjusted for age, sex, body mass index and hypertension.

cadjusted for age, sex, body mass index, diabetes and hypertension.

dadjusted for age, sex, body mass index and diabetes.

increased the risk and prevalence of diabetes and renal dysfunction, suggesting that blood Mn deficiency might be involved in the pathophysiological processes of diabetes and renal dysfunction.

$\mathrm{Mn}$ is stored primarily in skeletal bones and tissues rich in mitochondria. However, no existing biomarker can reliably determine the exact level of $\mathrm{Mn}$ accumulated in the body. Because there is a discrepancy in the half-life of $\mathrm{Mn}$ in the tissues and blood, Mn levels in red blood cells or whole blood are believed to be more reliable than plasma $\mathrm{Mn}$ for measuring $\mathrm{Mn}$ accumulation in the body [3]. For practical reasons, whole blood samples have been used as an exposure biomarker of $\mathrm{Mn}$ inhalation in most epidemiological studies [3] and were used accordingly in the present study to measure blood Mn.

The importance of $\mathrm{Mn}$ in animals was first reported in 1931, but there has been little evidence of its deficiency in humans [1]. In animal models, some of the abnormalities seen in Mn deficiency relate to skeletal abnormalities, changes in circulating HDL cholesterol, and reproductive failure [4]. Some studies have proposed that Mn may influence high glucose conditions. A relationship between $\mathrm{Mn}$ and pancreatic function was first seen in 1962 when the blood glucose level dropped in a diabetic patient orally administered Mn. It was postulated that Mn might accelerate cellular glucose uptake by potentiating insulin action and that $\mathrm{Mn}$ might act on the pancreas by stimulating the release of stored insulin into the bloodstream or by inhibiting the release of glucagon [10]. In addition, Baly et al. demonstrated that Mn deficiency resulted in decreased synthesis of pancreatic insulin, enhanced insulin degradation, and lowered insulin secretion in an Mn-deficient animal model [11]. It was also suggested that Mn deficiency could affect glucose transport and metabolism in adipose cells [12]. A recent experiment demonstrated in vitro and in vivo that $\mathrm{Mn}^{2+}$ supplementation lowered the risk of endothelial dysfunction in diabetes [13].

In the present study, we found a statistically significant relationship between blood $\mathrm{Mn}$ levels and diabetes in a representative Korean population. Blood Mn levels were lower in participants with diabetes but not in participants with ischemic heart disease or stroke. Furthermore, the prevalence of diabetes significantly increased in participants with blood Mn levels in the lowest quartile. The pathophysiological mechanism of these results remained unclear. However, the present findings were consistent with previous reports of hypoglycemia induced by lowdose $\mathrm{Mn}$ intake in diabetic patients, as well as changes in insulin metabolism in Mn-deficient animal models $[10,11,14]$. Further studies into the association of blood $\mathrm{Mn}$ and insulin metabolism or glucose control status (such as hemoglobin A1c) are needed.

Prior to this study, no clear relationship between $\mathrm{Mn}$ and diabetes had been found, and the few epidemiologic studies that addressed this issue in humans were controversial. With limited comparable data, one study into the general elderly population of the Czech Republic found no association between blood $\mathrm{Mn}$ and diabetes; instead, seniors with atherosclerosis had higher Mn levels than those without [15]. Three other studies linked low Mn levels with diabetes. Kazi et al. showed that diabetic patients of both genders in Pakistan had significantly lower blood and hair levels of Mn than those in the controls [16]. Ekmekcioglu et al. found decreased Mn levels in the whole blood and erythrocytes of diabetic individuals [17]. The most recent study revealed Mn deficiency in both type 1 and type 2 diabetic patients with respect to control subjects [18].

Many studies have focused on environmental Mn toxicity rather than on its deficiency and metabolism. Mn toxicity in humans primarily occurs as a consequence of chronic inhalation of high concentrations of airborne Mncontaining particles, linking symptoms of Mn toxicity mainly to miners as well as ferroalloy and battery manufacturing workers [5]. In Korea, Mn may be exposed occupationally or environmentally. Typical toxicity symptoms resemble those of idiopathic Parkinson's disease and include tremor, rigidity, bradykinesia, and posture instability. Patients may also display neuropsychological difficulties 
that include apathy and even psychosis as Mn affects the dopaminergic systems with a neuropathology that closely resembles Parkinson's disease [19]. Most studies have examined outcomes following relatively high-level acute or chronic exposure to Mn. Less is known about the effects of chronic exposure to lower levels of Mn or the threshold levels sufficient for altering cognitive and motor function [19].

Oxidative stress and inflammation play a major role in the progression of renal damage in chronic kidney disease (CKD). Mn is a potent antioxidant and cofactor of the enzyme MnSOD, which is the main antioxidant enzyme in the mitochondria responsible for protecting the cell from reactive oxygen species (ROS) by scavenging mitochondrial superoxides. Various studies using $\mathrm{Mn}^{2+}$ have linked its effects with the function and role of MnSOD. The results in this study showed a significant association of blood Mn level with the prevalence and risk of renal dysfunction after adjusting for diabetes and hypertension in logistic regression analysis, although not in linear regression. Because the KNAHNES was conducted in the general population of Koreans, we projected that including too small a size of subjects with moderate to advanced renal dysfunction may influence the statistical result. Thus, we cautiously postulated that subgroups with lower blood Mn levels may have lower MnSOD activities, possibly making them more sensitive to oxidative stress. Further studies are needed to verify this hypothesis.

High blood Mn levels were found, in this study, to be consistently associated with high systolic, diastolic, and mean arterial blood pressures after adjustment, although no differences in the prevalence of hypertension were found between the lower and higher blood Mn quartile subgroups. These results may have been conflicting as hypertension was partially dependent on self-reported data. Nevertheless, these findings were somewhat compatible with a previous study based on the KNHANES 2008, which reported that blood Mn levels may have been associated with an increased risk of hypertension [20]. The study assumed that chronic Mn accumulation in the mitochondria affected MnSOD activity, resulting in an abnormal response to the mechanism that blocked oxidative stress in the mitochondria and protected against endothelial dysfunction. The exact pathophysiology of Mn on blood pressure is unclear and remains to be elucidated.

This study had some limitations. First, it was crosssectional, making it difficult to establish a cause-andeffect relationship. Diabetes appeared to have caused lower Mn based on data showing low stores of albumin and other binding proteins in diabetic patients [21], suggesting that $\mathrm{Mn}$ and diabetes may affect each other. Further analysis with a longer follow-up period is needed to support the clinical importance of blood $\mathrm{Mn}$ levels in diabetes. Second, our regression models were not fully adjusted for potential confounders, such as serum iron or albumin levels, which are the main Mn-binding proteins in the blood. Furthermore, we did not adjust for other metals with the same valence states as Mn, including copper and calcium, which may affect blood Mn levels and other chronic diseases. There may have been participants with chronic diseases without Mn readings, which may explain the lack of a significant association between blood Mn levels and ischemic heart disease or stroke. Last, insufficient information was available to specify whether the diabetes subgroups were type I or II. Despite these limitations, the study presented solid findings as it was performed on a representative sample of the general Korean population with strict quality control of the study procedures of the KNHANES.

\section{Conclusion}

The prevalence of diabetes and renal dysfunction decreased in participants with high blood Mn levels, suggesting that blood Mn may have some role in glucose homeostasis and renal function. Further studies are needed to determine any protective effects of $\mathrm{Mn}$ on diabetes and renal dysfunction.

\section{Competing interests}

The authors declare that they have no competing interests.

\section{Authors' contributions}

SJS had full access to all the data in the study and took responsibility for the integrity of the data and accuracy of the data analysis. Study concept and design: SJS, SC, and JHC. Acquisition of data: SJS, ESK, HEY, and SJK. Statistical analysis and interpretation of data: SJS, SC, and JHC. Draft of the manuscript: SJS and ESK. Critical revision of the manuscript for important intellectual content: CWP and YSC. All authors read and approved the final manuscript.

\section{Author details}

${ }^{1}$ Department of Internal Medicine, College of Medicine, The Catholic University of Korea, 222 Banpo-daero, Seoul 137-701, Republic of Korea. 2Division of Nephrology, The Catholic University of Korea Yeouido St. Mary's Hospital, 10, 63-ro, Yeongdeungpo-gu, Seoul 150-713, Republic of Korea. ${ }^{3}$ Division of Nephrology, The Catholic University of Korea Incheon St. Mary's Hospital, 56, Dongsu-ro, Bupyeong-gu, Incheon 403-720, Republic of Korea. ${ }^{4}$ Department of Statistics, The Graduate School of Ewha Womans University, 52, Ewhayeodae-gil, Seodaemun-gu, Seoul 120-750, Republic of Korea. ${ }^{5}$ Division of Nephrology, The Catholic University of Korea Seoul St. Mary's Hospital, 222, Banpo-daero, Seoul 137-701, Republic of Korea.

Received: 5 July 2013 Accepted: 5 March 2014

Published: 8 March 2014

\section{References}

1. Aschner $J$, Aschner M: Nutritional aspects of manganese homeostasis. Mol Aspects Med 2005, 26(4-5):353-362.

2. Malecki EA, Huttner DL, Greger JL: Manganese status, gut endogenous losses of manganese, and antioxidant enzyme activity in rats fed varying levels of manganese and fat. Biol Trace Elem Res 1994, 42(1):17-29.

3. Rucker $D$, Thadhani $R$, Tonelli M: Trace element status in hemodialysis patients. Seminars in dialysis 2010, 23(4):389-395.

4. Greger JL: Nutrition versus toxicology of manganese in humans: evaluation of potential biomarkers. Neurotoxicology 1999, 20(2-3):205-212.

5. Manganese deficiency in humans: fact or fiction? Nutrition reviews 1988, 46(10):348-352.

6. HaMai D, Rinderknecht AL, Guo-Sharman K, Kleinman MT, Bondy SC: Decreased expression of inflammation-related genes following inhalation exposure to manganese. Neurotoxicology 2006, 27(3):395-401. 
7. Malecki EA, Greger JL: Manganese protects against heart mitochondrial lipid peroxidation in rats fed high levels of polyunsaturated fatty acids. J Nutr 1996, 126(1):27-33.

8. Levey AS, Stevens LA, Schmid CH, Zhang YL, Castro AF 3rd, Feldman HI, Kusek JW, Eggers P, Van Lente F, Greene T, Coresh J: A new equation to estimate glomerular filtration rate. Ann Intern Med 2009, 150(9):604-612.

9. Greenberg A: Urinalysis. In Primer on Kidney Diseases. 5th edition. Edited by Greenberg A, Cheung AK, Coffman TM, Falk RJ, Jennette JC. Philadelphia: Saunders; 2009:24-32.

10. Rubenstein AH, Levin NW, Elliott GA: Manganese-induced hypoglycaemia. Lancet 1962, 2(7270):1348-1351.

11. Baly DL, Curry DL, Keen CL, Hurley LS: Dynamics of insulin and glucagon release in rats: influence of dietary manganese. Endocrinology 1985, 116(5):1734-1740.

12. Baly DL, Schneiderman JS, Garcia-Welsh AL: Effect of manganese deficiency on insulin binding, glucose transport and metabolism in rat adipocytes. J Nutr 1990, 120(9):1075-1079.

13. Burlet $E$, Jain SK: Manganese supplementation reduces high glucose-induced monocyte adhesion to endothelial cells and endothelial dysfunction in Zucker diabetic fatty rats. $J$ Biol Chem 2013, 288(9):6409-6416.

14. Baly DL, Curry DL, Keen CL, Hurley LS: Effect of manganese deficiency on insulin secretion and carbohydrate homeostasis in rats. J Nutr 1984, 114(8):1438-1446.

15. Rambouskova J, Krskova A, Slavikova M, Cejchanova M, Wranova K, Prochazka B, Cerna M: Trace elements in the blood of institutionalized elderly in the Czech Republic. Arch Gerontol Geriatr 2013, 56(2):389-394.

16. Kazi TG, Afridi HI, Kazi N, Jamali MK, Arain MB, Jalbani N, Kandhro GA: Copper, chromium, manganese, iron, nickel, and zinc levels in biological samples of diabetes mellitus patients. Biol Trace Elem Res 2008, 122(1):1-18.

17. Ekmekcioglu C, Prohaska C, Pomazal K, Steffan I, Schernthaner G, Marktl W: Concentrations of seven trace elements in different hematological matrices in patients with type 2 diabetes as compared to healthy controls. Biol Trace Elem Res 2001, 79(3):205-219.

18. Forte G, Bocca B, Peruzzu A, Tolu F, Asara Y, Farace C, Oggiano R, Madeddu R: Blood metals concentration in type 1 and type 2 diabetics. Biol Trace Elem Res 2013, 156(1-3):79-90

19. Aschner M, Guilarte TR, Schneider JS, Zheng W: Manganese: recent advances in understanding its transport and neurotoxicity. Toxicol Appl Pharmacol 2007, 221(2):131-147.

20. Lee BK, Kim Y: Relationship between blood manganese and blood pressure in the Korean general population according to KNHANES 2008. Environ Res 2011, 111(6):797-803.

21. Folsom AR, Ma J, Eckfeldt JH, Nieto FJ, Metcalf PA, Barnes RW: Low serum albumin. Association with diabetes mellitus and other cardiovascular risk factors but not with prevalent cardiovascular disease or carotid artery intima-media thickness. The Atherosclerosis Risk in Communities (ARIC) Study Investigators. Ann Epidemiol 1995, 5(3):186-191.

doi:10.1186/1472-6823-14-24

Cite this article as: Koh et al: Association of blood manganese level with diabetes and renal dysfunction: a cross-sectional study of the Korean general population. BMC Endocrine Disorders 2014 14:24

\section{Submit your next manuscript to BioMed Central and take full advantage of:}

- Convenient online submission

- Thorough peer review

- No space constraints or color figure charges

- Immediate publication on acceptance

- Inclusion in PubMed, CAS, Scopus and Google Scholar

- Research which is freely available for redistribution

Submit your manuscript at www.biomedcentral.com/submit 